\title{
Prenatal Stress Alters the Development of Socioemotional Behavior and Amygdala Neuron Excitability in Rats
}

\author{
David E Ehrlich' and Donald G Rainnie ${ }^{*, 1}$ \\ 'Division of Behavioral Neuroscience and Psychiatric Disorders, Department of Psychiatry and Behavioral Sciences, Yerkes Research Center, Emory \\ University School of Medicine, Atlanta, GA, USA
}

\begin{abstract}
Prenatal stress (PS) is a risk factor for neurodevelopmental disorders with diverse ages of onset and socioemotional symptoms. Some PS-linked disorders involve characteristic social deficits, such as autism spectrum disorders and schizophrenia, but PS also promotes anxiety disorders. We propose the diversity of symptoms following PS arises from perturbations to early brain development. To this end, we characterized the effects of PS on the developmental trajectory of physiology of the amygdala, a late-developing center for socioemotional control. We found that PS dampened socioemotional behavior and reduced amygdala neuron excitability in offspring during infancy (at postnatal days (P) I0, 14, I7 and 21), preadolescence (day 28), and adulthood (day 60). PS offspring in infancy produced fewer isolation-induced vocalizations and in adulthood exhibited less anxiety-like behavior and deficits in social interaction. PS neurons had a more hyperpolarized resting membrane potential from infancy to adulthood and produced fewer action potentials. Moreover, adult amygdala neurons from PS animals expressed larger action potential afterhyperpolarizations and $\mathrm{H}$-current relative to controls, further limiting excitability. Our results suggest that PS can suppress socioemotional behavior throughout development and produce age-specific alterations to amygdala physiology.

Neuropsychopharmacology (2015) 40, 2I35-2I45; doi:I0.I038/npp.20I5.55; published online 25 March 2015
\end{abstract}

\section{INTRODUCTION}

Prenatal stress (PS) is a risk factor for neurodevelopmental disorders that emerge at distinct ages and whose symptoms span multiple socioemotional domains. For instance, PS is linked to disorders with characteristic social deficits that typically emerge in children (autism spectrum disorders and attention-deficit/hyperactivity disorder) and adolescence (schizophrenia) (Khashan et al, 2008; Kinney et al, 2008; Ronald et al, 2010). These disorders frequently present without comorbid anxiety, but PS is also a risk factor for anxiety disorders (Monk et al, 2012). This diversity of outcomes is also reflected in preclinical studies of PS, which often fail to recapitulate social deficits or heightened anxiety (Estanislau and Morato, 2006; Mairesse et al, 2007; Schulz et al, 2011). Given the variability of PS effects on socioemotional behavior, offspring outcomes may be determined by the developmental timing when deficits emerge (Tottenham and Sheridan, 2009; Blackford and Pine, 2012; Callaghan et al, 2013). Characterizing trajectories of the brain development may help elucidate the mechanisms by which PS

\footnotetext{
*Correspondence: Dr DG Rainnie, Division of Behavioral Neuroscience and Psychiatric Disorders, Department of Psychiatry and Behavioral Sciences, Yerkes Research Center, Emory University School of Medicine, Atlanta, GA 30329, USA, Tel: +404 712 9714, Fax: +404 727 3233, E-mail: drainni@emory.edu

Received I8 November 20 14; revised I3 February 2015; accepted 23 February 2015; accepted article preview online 26 February 2015
}

confers risk for a diversity of neurodevelopmental disorders and provide potential targets for intervention.

A late-developing brain center for socioemotional control (Giedd et al, 1996; Deoni et al, 2011), the amygdala is implicated in the pathophysiology of neurodevelopmental psychiatric disorders (Quirk and Gehlert, 2003; Amaral et al, 2008; Benes, 2010; Felix-Ortiz and Tye, 2014). Adult amygdala function and emotional behavior are altered by PS (Weinstock, 2008; Sadler et al, 2011), and several studies have identified effects of PS on the developing amygdala (Kraszpulski et al, 2006; Laloux et al, 2012). However, no study to date has described how PS influences the developmental trajectory of amygdala function or characterized the effects of PS on amygdala neurophysiology, regardless of age.

Studies in rats have illustrated that amygdala development occurs pre- and postnatally. Emerging during the third week of gestation, the basolateral nucleus of the amygdala (BLA) is a hub that receives multimodal sensory input and coordinates behaviorally relevant output (Bayer et al, 1993; Berdel et al, 1997a; LeDoux, 2007; Walker and Davis, 2008). Neurons in the BLA mature prominently throughout infancy and adolescence (Berdel et al, 1997b; Ryan et al, 2014), when amygdala-dependent emotional behavior also undergoes marked development (Landers and Sullivan, 2012; King et al, 2014). During this period, fold changes are observed in the intrinsic membrane properties, action potential (AP) kinetics, and synaptic and voltage-gated currents of BLA principal neurons (Ehrlich et al, 2012; Ehrlich et al, 2013 ), which comprise $\sim 85 \%$ of neurons in the nucleus 
(McDonald, 1985). We propose that heightened plasticity of amygdala neurons during infancy and adolescence contributes to the emergence of deficits following PS.

We tested the hypothesis that PS influences BLA neurophysiology in an age-specific manner, with effects emerging postnatally. Given that amygdala gene expression and socioemotional behavior are influenced by late-term PS (Laloux et al, 2012) and chronic unpredictable shock stress (Hazra et al, 2012), we exposed pregnant rats to unpredictable shocks from gestational days (G) 17-20. We measured the effects of PS on male offspring at P10, 14, 17, 21, and 28, as well as young adults at P60. In infancy we quantified ultrasonic vocalizations (USVs) as a measure of socioemotional behavior, and in adults we measured anxiety-like behavior and sociability. Across all ages, we characterized PS-induced changes to intrinsic membrane properties of BLA principal neurons in acute brain slices using whole-cell patch clamp. We found that PS induced a constellation of changes to amygdala neurophysiology that varied in age of onset and duration. These alterations generally served to reduce amygdala excitability, and may thereby explain the dampened socioemotional behavior observed throughout development following PS.

\section{MATERIALS AND METHODS}

All experimental protocols strictly conform to the Guidelines for the Care and Use of Laboratory Animals of the National Institutes of Health, and were approved by the Emory University Institutional Animal Care and Use Committee.

\begin{abstract}
Animals
Males born in-house to time-mated, Sprague-Dawley rats (G4 on arrival, Charles River, Wilmington, MA) were used in all experiments. Rats were maintained on a 12-12-h lightdark cycle and given access to food and water ad libitum. Pups were housed with their dam before weaning on P22 or P23, with the day of birth considered P1. After weaning, rats were isolated by sex and housed three to four per cage. Animal ages are attributed to a single day for brevity, but labels describe developmental windows as follows: 'P10' for $\mathrm{P} 10-11$, 'P14' for P13-14, 'P17' for P17-18, 'P21' for P21-22, 'P28' for P27-30, and 'P60' for P60-70.
\end{abstract}

\section{Prenatal Stress}

An unpredictable shock stress paradigm was applied as previously described (Hazra et al, 2012) to pregnant dams as follows. On each of G17-20, dams were placed in a conditioning chamber measuring $60 \times 34 \times 26 \mathrm{~cm}$, with aluminum and polycarbonate walls (Lafayette Instruments, Lafayette, IN). The floor of the chamber, composed of 0.4-cm-diameter stainless steel bars, conducted the electric shock. Dams in the PS group were allowed to habituate to the chamber for $5 \mathrm{~min}$ and then received two 8-min periods of shocks separated by an 8-min period without shocks (Supplementary Figure S1A). Each shock period consisted of eight pseudo-randomly applied foot-shocks $(0.5 \mathrm{~s}, 0.5 \mathrm{~mA}$, 30-90 s variable inter-shock interval). Non-stressed control dams received the same handling procedures as the PS group and were placed in the shock chamber for the same duration without shocks applied. During each 5-min habituation block, the behavior of some dams was recorded with an overhead video camera and analyzed offline for freezing with FreezeScan (Clever Sys Inc., Reston, VA). After exposure to the chamber on G20, dams were returned to their home cages and left to give birth and rear pups normally.

\section{Behavioral Testing}

Socioemotional behavior was measured in P7 and P17 offspring by isolation-induced USVs, based on the protocol described by Hofer et al, (2002). At P60, anxiety-like behavior was measured in the elevated plus maze (EPM) and open-field test, and sociability was measured in the social choice test. For details on these behavioral paradigms, please refer to the Supplementary Materials and Methods.

\section{Electrophysiology}

Tissue preparation. Offspring of stress-exposed and control dams were used for electrophysiological studies. Slices were prepared from rats at various ages as previously described (Ehrlich et al, 2012). Rats were decapitated under isoflurane anesthesia (Fisher Scientific, Hanover Park, IL) at P14 and older or without anesthesia before P14. Brains were rapidly removed and immersed in ice-cold 95\% oxygen-5\% carbon dioxide-perfused 'cutting solution' with the following composition (in mM): $130 \mathrm{NaCl}, 30 \mathrm{NaHCO}_{3}, 3.50 \mathrm{KCl}, 1.10$ $\mathrm{KH}_{2} \mathrm{PO}_{4}, 6.0 \mathrm{MgCl}_{2}, 1.0 \mathrm{CaCl}_{2}, 10$ glucose, 0.4 ascorbate, 0.8 thiourea, 2.0 sodium pyruvate, and 2.0 kynurenic acid. Coronal slices containing the BLA were cut at a thickness of 300-350 $\mu \mathrm{m}$ with a Leica VTS-1000 vibrating blade microtome (Leica Microsystems, Bannockburn, IL). Slices were incubated for $1 \mathrm{~h}$ in oxygenated cutting solution at $32^{\circ} \mathrm{C}$ before being transferred to regular artificial cerebrospinal fluid (ACSF) containing (in mM) $130 \mathrm{NaCl}, 30 \mathrm{NaHCO}_{3}$, $3.50 \mathrm{KCl}, 1.10 \mathrm{KH}_{2} \mathrm{PO}_{4}, 1.30 \mathrm{MgCl}_{2}, 2.50 \mathrm{CaCl}_{2}, 10$ glucose, 0.4 ascorbate, 0.8 thiourea, and 2.0 sodium pyruvate.

Whole-cell patch clamp. Individual slices were transferred to a recording chamber mounted on the fixed stage of a Leica DMLFS microscope (Leica Microsystems) and maintained fully submerged and continuously perfused with oxygenated $32{ }^{\circ} \mathrm{C} \mathrm{ACSF}$ at a flow rate of $1-2 \mathrm{ml} / \mathrm{min}$. The BLA was identified under $\times 10$ magnification, and individual BLA neurons were identified at $\times 40$ with differential interference contrast optics and infrared illumination with an infraredsensitive CCD camera (Orca ER, Hamamatsu, Tokyo, Japan). Patch pipettes of 4-6 M $\Omega$ were pulled from borosilicate glass. Two patch electrode solutions were used, one based on potassium gluconate for current-clamp recordings and one based on cesium gluconate for voltage-clamp recordings. The potassium gluconate patch solution had the following composition (in $\mathrm{mM}$ ): 140 potassium gluconate, $2 \mathrm{KCl}, 10$ HEPES, $3 \mathrm{MgCl}_{2}, 2 \mathrm{~K}$-ATP, $0.2 \mathrm{Na}-\mathrm{GTP}$, and 5 phosphocreatine, was titrated to $\mathrm{pH} 7.3$ with $\mathrm{KOH}$, and was 290 mosM. The cesium gluconate patch solution had the following composition (in $\mathrm{mM}$ ): $131 \mathrm{CsOH}, 131$ gluconate, $10 \mathrm{HEPES}, 2 \mathrm{CaCl}_{2}, 10$ glucose, $10 \mathrm{EGTA}, 5 \mathrm{Mg}$-ATP, and $0.4 \mathrm{Na}-\mathrm{GTP}$, was titrated to $\mathrm{pH} 7.3$ with gluconate, and was 270 mosM. For details on data acquisition and 
a
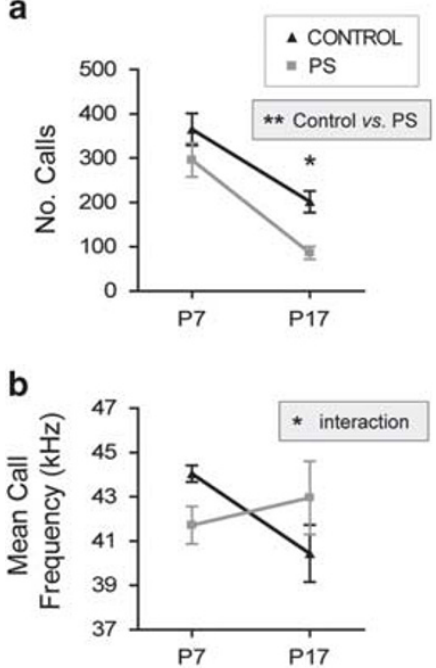

C

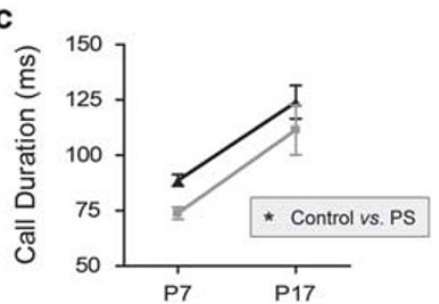

d

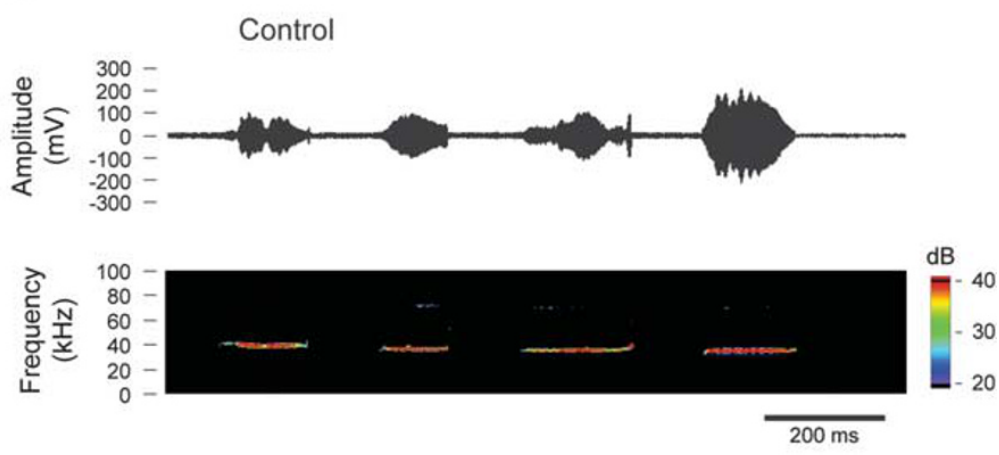

Prenatal Stress
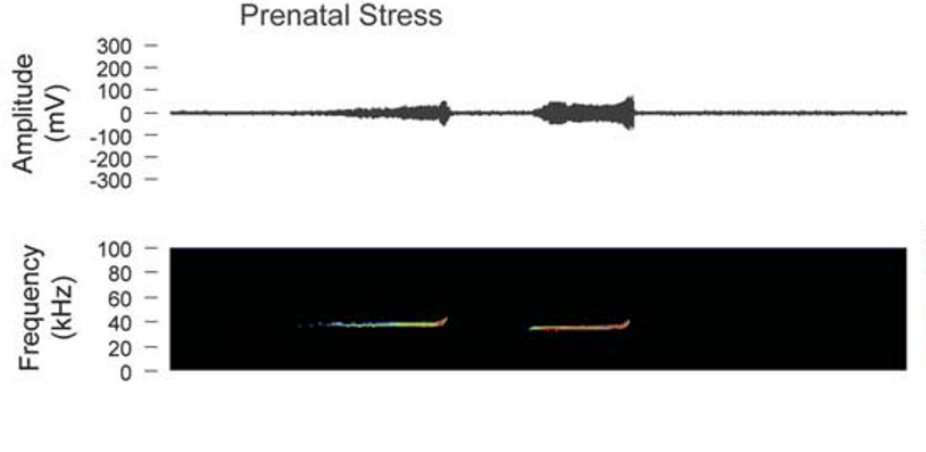

Figure I PS reduced isolation-induced vocalizations in infant offspring. (a) Total number of pup calls during 5-min isolation was significantly reduced with age and in PS animals at PI7 relative to controls. (b) The mean frequency of pup calls is plotted for control and PS offspring at P7 and I7. (c) Pup call duration increased with age and was significantly shorter for PS animals. (d) Windows depicting representative pup calls for control and PS offspring at PI7, with call envelope and spectrogram illustrating reduced call rate and amplitude for PS animals.

electrophysiological data analysis, please refer to Supplementary Materials and Methods.

\section{Sampling and Statistics}

Regarding all PS and control offspring, behavioral data were collected on the EPM from 37 adult males (18 rats from five PS litters and 19 rats from five control litters), 27 of which were also tested 2 days prior using the open-field test. A distinct population of 44 adult male rats (20 rats from six PS litters and 24 rats from six control litters) was tested in the sociability and social novelty tests. In addition, 27 rats from five PS litters and 32 rats from five control litters were tested at P7 and P17 for isolation-induced USVs. Some rats tested for USVs were later utilized for electrophysiological studies at P60, but otherwise the animals providing tissue for slice recordings were behaviorally naive. Electrophysiological data were collected from 445 BLA neurons from 85 male rats on P10, 14, 17, 21, 28, and 60, representing 29 distinct litters (at least four litters at each time point/group). Neurons were divided into different data sets based on the recording configuration and patch solution, and a total of 186 neurons were recorded in current-clamp mode and 259 neurons in voltage-clamp mode. Neurons were recorded in both configurations from most animals included in the study. A total of 221 neurons were recorded from PS animals and 224 recorded from controls.
All data are plotted as mean \pm SEM unless otherwise noted. The following annotations are used to ascribe statistical significance: ${ }^{\star} P<0.05 ;{ }^{\star \star} P<0.01 ;{ }^{\star * \star} P<0.001 ;{ }^{\star * *} P<0.0001$. For details on statistical tests employed, please refer to Supplementary Materials and Methods.

\section{RESULTS}

We first assessed behavioral effects of the PS paradigm directly on pregnant dams. PS dams reliably displayed freezing in the shock chamber before foot-shock presentation on days 2-4 of the PS paradigm (Supplementary Figure S1B; two-way ANOVA, interaction effect: $\mathrm{F}_{3,41}=51.15, \quad P<0.0001$; $6 \leqslant n \leqslant 7)$.

\section{PS Reduced Anxiety-Like Behavior in Infant and Adult Offspring}

We next tested how PS influenced the development of offspring emotional behavior. As illustrated in Figure 1, infant offspring of dams exposed to PS produced fewer isolationinduced USVs, a measure of anxiety-like behavior (Knutson et al, 2002). With age, pups in both groups produced fewer USVs during $5 \mathrm{~min}$ of isolation from their dams (Figure 1a and d; three-way ANOVA, main effect of age: $\mathrm{F}_{1,91}=34.46$, $P<0.0001 ; 3 \leqslant n \leqslant 9$ pups per litter for five PS and five control litters), and by P17 an effect of PS on USV number 

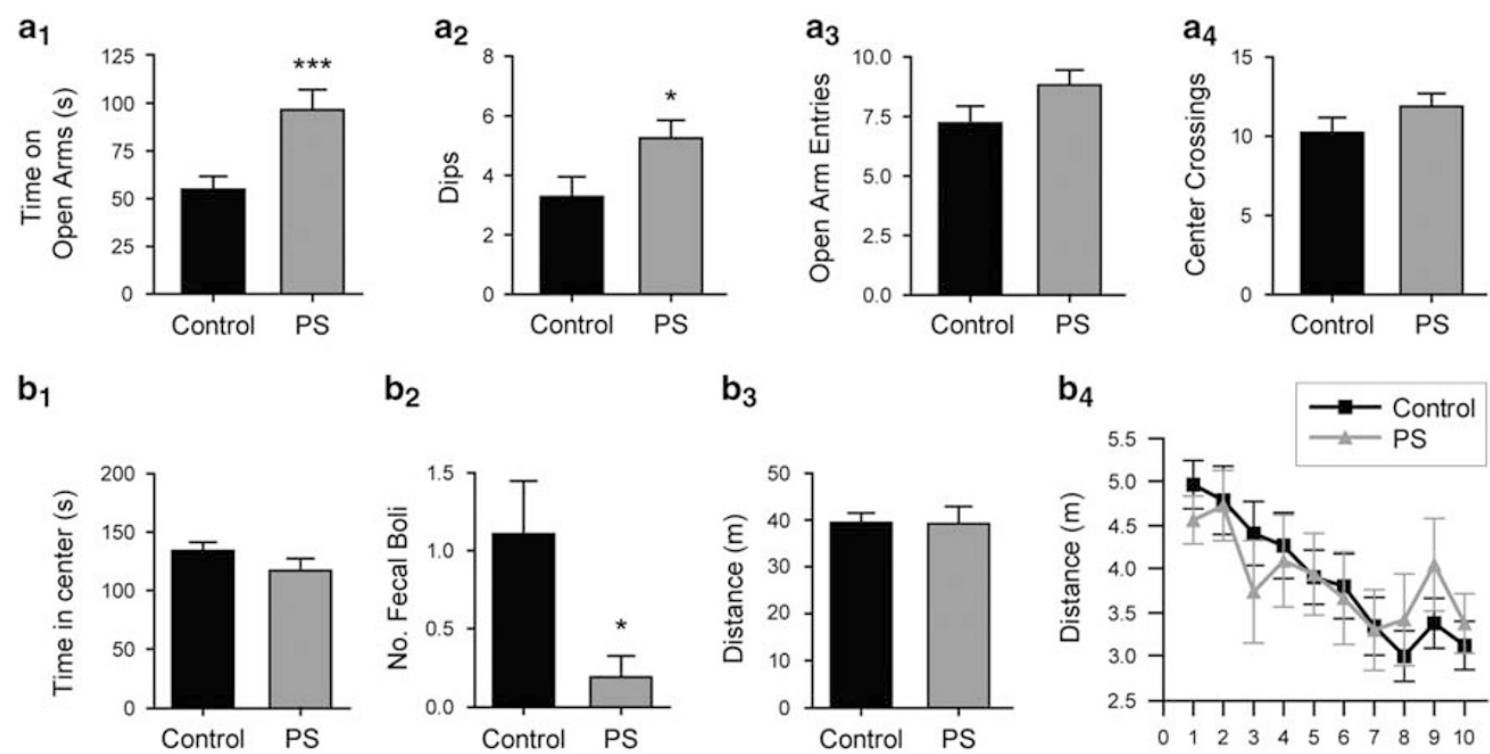

$b_{2}$

$b_{3}$
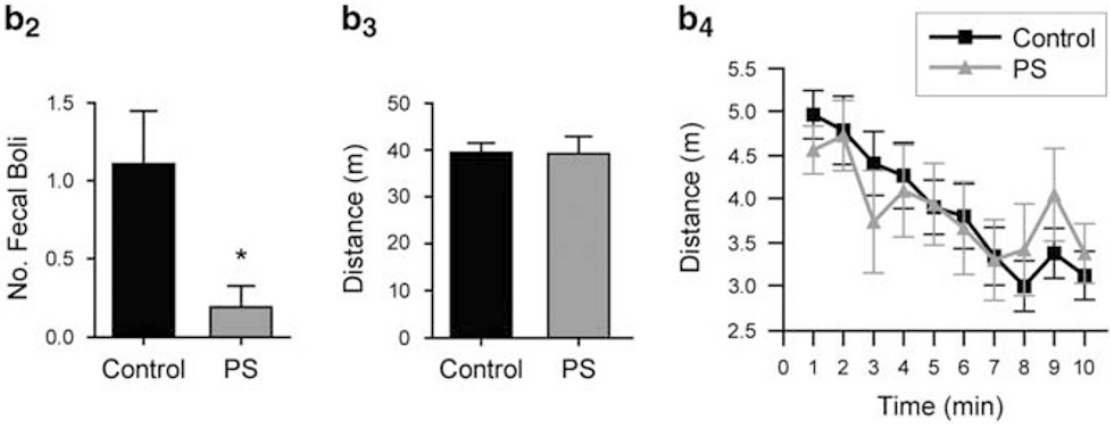

$\mathrm{c}_{1}$

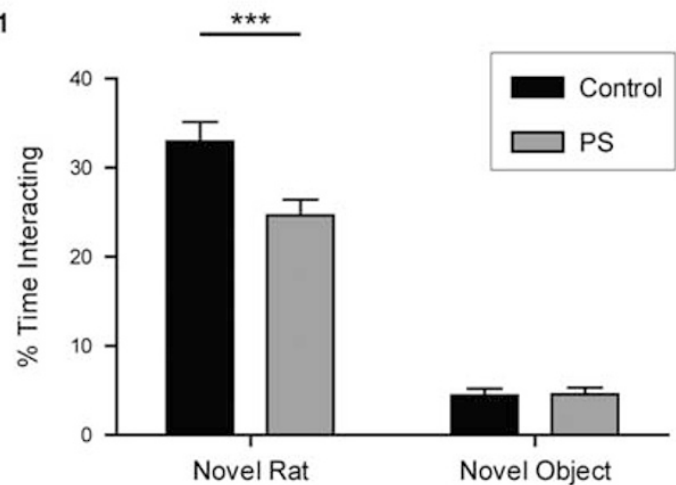

$\mathrm{c}_{2}$

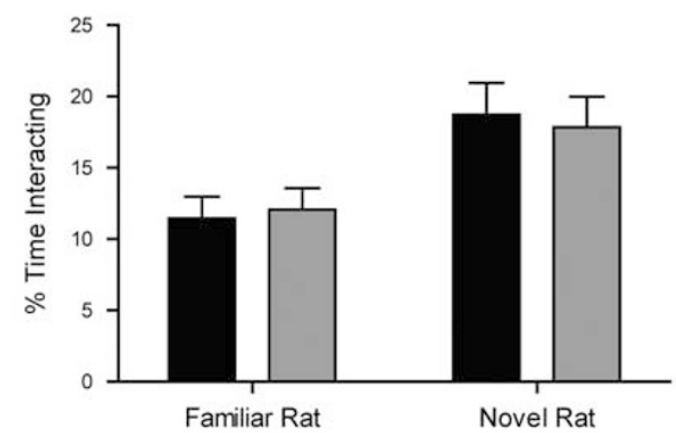

Figure 2 PS reduced socioemotional behavior in adult offspring. ( $\left.a_{1}\right)$ Time spent on open arms of the elevated plus maze was significantly greater in PS animals compared with non-stressed controls. $\left(a_{2}\right)$ PS rats made significantly more head dips on the plus maze than controls. $\left(a_{3}, a_{4}\right)$ There was no effect of PS on open-arm entries $\left(a_{3}\right)$ or locomotor activity on the plus maze, as measured by center crossings $\left(a_{4}\right)$. $\left(b_{1}\right)$ Time spent in center of the open-field did not significantly differ between adult PS offspring and controls. $\left(b_{2}\right)$ PS rats produced fewer fecal boli than non-stressed controls during the open-field test. ( $b_{3}$, $b_{4}$ ) No effect of PS in the open-field test was observed on total distance traveled $\left(b_{3}\right)$ or distance traveled during any I-min bin ( $\left.b_{4}\right)$. ( $\left.c_{1}\right)$ Adult PS offspring showed less preference than controls for a novel conspecific during a 5-min sociability test. $\left(C_{2}\right)$ PS offspring and controls spent comparable time interacting with a familiar conspecific and a novel conspecific during a 5-min social novelty test.

emerged $(201.8 \pm 24.5$ USVs for 28 control pups $v s$ $86.4 \pm 14.3$ USVs for 24 PS pups; Bonferroni post-test, $P<0.05$; main effect of PS: $\left.\mathrm{F}_{1,91}=12.46, P<0.001\right)$. The quality of USVs was also altered by PS, both in terms of pitch (Figure $1 \mathrm{~b}$ ) and duration (Figure 1c). The mean frequency of USVs decreased with age in control pups $(44.0 \pm 0.4 \mathrm{kHz}$ for 32 P7 pups to $40.4 \pm 1.3 \mathrm{kHz}$ for 27 pups at P17), but increased with age in PS pups $(41.7 \pm 0.8 \mathrm{kHz}$ for 27 pups at P7 to $43.0 \pm 1.7 \mathrm{kHz}$ for 22 pups at P17; three-way ANOVA, interaction effect of PS and age: $\left.\mathrm{F}_{1,88}=8.18, P<0.01\right)$. Across both time points, USVs produced by control pups were of longer duration than those produced by PS pups (three-way ANOVA, main effect of PS: $\mathrm{F}_{1,88}=5.94, P<0.05$; main effect of age: $F_{1,88}=32.72, P<0.0001$; main effect of litter: $\left.\mathrm{F}_{8,88}=2.62, P<0.05\right)$. In addition, USVs from PS pups had qualitatively smaller amplitudes (Figure 1d).
The apparent anxiolytic effect of PS was also observed in adult offspring, as measured in the EPM (Figure 2a). Adult offspring of PS dams spent significantly more time on the open arms of the plus maze during the 5-min test (Figure $2 \mathrm{a}_{1} ; 54.8 \pm 6.9 \mathrm{~s}$ for 18 controls $v s 96.5 \pm 10.6 \mathrm{~s}$ for 16 PS adults; two-way ANOVA, main effect of PS: $\mathrm{F}_{1,24}=18.13$, $P<0.001$; main effect of litter: $\mathrm{F}_{8,24}=2.46, P<\mathrm{L} 0.05$; $2 \leqslant n \leqslant 8$ pups per litter for five PS and five control litters). Compared with controls, PS rats also performed significantly more head dips on the plus maze, also indicating less anxiety (Figure $2 \mathrm{a}_{2} ; 3.3 \pm 0.7$ dips for controls $v s 5.3 \pm 0.6$ dips for PS adults; Mann-Whitney $U$-test, $\left.U_{32}=85, P<0.05\right)$. No group difference was observed in terms of open-arm entries (Figure $2 \mathrm{a}_{3} ; 7.2 \pm 0.7$ entries for controls $v s 8.8 \pm 0.6$ entries for PS adults; $U_{32}=96.5, P>0.05$ ), indicating rats from both groups entered the open arms with the same frequency, but bouts lasted longer for offspring of PS dams. There was no 
impact of PS on locomotor activity as assessed by center crossings (Figure $2 \mathrm{a}_{4} ; 10.2 \pm 1.0$ crossings for controls $v s$ $11.9 \pm 0.8$ crossings for PS adults; $\left.U_{32}=112.5, P>0.05\right)$.

In contrast, no effect of PS on anxiety-like behavior of adult offspring was detected in the open-field test, as determined by motoric behavior (Figure 2b). PS and control offspring spent comparable amounts of time in the center of the open field during the 10-min test (Figure 2 $\mathrm{b}_{1}$; $133.9 \pm 7.5 \mathrm{~s}$ for 18 controls $v s 117.1 \pm 10.4 \mathrm{~s}$ for 18 PS adults; two-way ANOVA, main effect of PS: $F_{1,26}=2.25, P>0.05$; main effect of litter: $\mathrm{F}_{8,26}=4.58, P<0.01 ; 2 \leqslant n \leqslant 8$ pups per litter for three PS and four control litters). However, PS exerted an anxiolytic effect in the open field as measured by the production of fecal boli. PS offspring produce fewer fecal boli during the test (Figure $2 b_{2} ; 1.11 \pm 0.34$ boli for controls vs $0.19 \pm 0.14$ boli for PS adults; $\left.U_{33}=102, P<0.05\right)$. Similar to on the EPM, there was no effect of PS on locomotor activity in the open field, measured either by total distance traveled (Figure 2b $b_{3} ; 39.3 \pm 2.3 \mathrm{~m}$ for 16 controls $v s$ $39.2 \pm 3.8 \mathrm{~m}$ for $11 \mathrm{PS}$ adults; two-way ANOVA with factors of PS and litter, main effect of PS: $\left.\mathrm{F}_{1,20}=0.696, P>0.05\right)$ or distance traveled per minute (Figure $2 \mathrm{~b}_{4}$; repeated measures two-way ANOVA, main effect of PS: $\left.\mathrm{F}_{1,25}=0.001, P>0.05\right)$.

\section{PS Reduced Sociability of Adult Offspring}

The effects of PS on sociability and social novelty preference were then measured in adult offspring in the social choice test (Figure 2c). PS offspring exhibited a weaker preference for social to non-social stimuli, spending significantly less time interacting with the conspecific (CON) during the 5min sociability test (Figure 2 $c_{1}$; Bonferroni post-test of duration with $\mathrm{CON}, P<0.001$; controls: $123.0 \pm 8.7 \mathrm{~s}$ with CON vs $16.4 \pm 3.2 \mathrm{~s}$ with object, $n=23$; PS adults: $92.5 \pm 6.4 \mathrm{~s}$ with CON vs $17.2 \pm 2.8 \mathrm{~s}$ with object, $n=20$; repeated measures two-way ANOVA, main effect of stress: $\mathrm{F}_{1,41}=8.98, P<0.01$; interaction effect: $\mathrm{F}_{1,41}=5.37, P<0.05$; $20 \leqslant n \leqslant 23)$. Furthermore, PS offspring exhibited greater latencies to interact with the CON $(38.7 \pm 11.3 \mathrm{~s}$ for 20 PS offspring $v s 25.9 \pm 5.7 \mathrm{~s}$ for 24 control offspring; two-way ANOVA, main effect of PS: $\mathrm{F}_{1,31}=5.21, P<0.05$; main effect of litter: $\mathrm{F}_{10,31}=6.7, P<0.0001 ; 2 \leqslant n \leqslant 7$ pups per litter for five PS and six control litters). However, no effect of PS was observed in the social novelty test, indicating social recognition was intact, despite decreased preference for social stimuli. PS and control rats both exhibited a preference for a novel over familiar CON (Figure $2 \mathrm{c}_{2}$; controls: $34.2 \pm 4.7 \mathrm{~s}$ with familiar CON vs $56.0 \pm 6.9$ s with novel CON; PS adults: $36.3 \pm 4.4 \mathrm{~s}$ with familiar CON vs $53.6 \pm 6.3 \mathrm{~s}$ with novel $\mathrm{CON}$; repeated measures two-way ANOVA, main effect of CON: $\mathrm{F}_{1,41}=10.10, P<0.01$; main effect of PS: $\mathrm{F}_{1,41}=0.001$, $P>0.05)$.

\section{No Effect of PS on Body Mass or Litter Size}

The trajectory of offspring growth was not altered by PS, with no significant effect on body mass (Supplementary Figure S2; three-way ANOVA, main effect of PS: $\mathrm{F}_{1,38}=0.21$, $P<0.05$; main effect of age: $\mathrm{F}_{5,38}=2218.12, P<0.0001$; for $\mathrm{P} 10-28,4 \leqslant N \leqslant 6$; for $\mathrm{P} 60,22 \leqslant n \leqslant 26$ ). However, body mass tended to be greater in PS offspring from P14 onwards, an effect most pronounced at P60 $(355.3 \pm 13.3 \mathrm{~g}$ for 26 control adults vs $400.4 \pm 9.0 \mathrm{~g}$ for $22 \mathrm{PS}$ adults). There was also no effect of PS on litter size (data not shown; $11.0 \pm 0.6$ pups per PS litter $v s 12.0 \pm 0.4$ pups per control litter, Student's $t$-test, $P>0.05, n=21)$.

\section{PS Altered Membrane Properties of Developing and Mature BLA Principal Neurons}

Having observed that PS suppressed anxiety-like and social behavior, both of which involve activation of amygdala neurons, we next characterized the effects of PS on the developmental trajectory of BLA principal neuron electrophysiology. We first addressed the effect of PS on maturation of intrinsic membrane properties (Figure 3). We previously reported the trajectory for typical development of input resistance $\left(R_{\text {in }}\right)$ and membrane time constant, which decrease by more than threefold over the first 3 postnatal weeks (Ehrlich et al, 2012). Here we found no effect of PS on the trajectory for $R_{\text {in }}$ (Figure $3 \mathrm{a}_{1}$; two-way ANOVA, main effect of PS: $\left.\mathrm{F}_{1,161}=0.86, P>0.05 ; 7 \leqslant n \leqslant 19\right)$ or membrane time constant (Figure $3 \mathrm{a}_{2}$; main effect of PS: $\mathrm{F}_{1,159}=0.18, P>0.05$ ). In contrast, PS did influence the resting membrane potential (RMP, Figure $3 a_{3}$ ). As expected, we found a significant effect of age, with RMP in control pups of $-55.3 \pm 1.0 \mathrm{mV}$ at P10 that became more hyperpolarized by $\mathrm{P} 17$, reaching the mature value of $-62.9 \pm 0.8$ (two-way ANOVA, main effect of age: $\left.\mathrm{F}_{5,148}=17.3, P<0.0001\right)$. Across all ages, the RMP was more hyperpolarized for neurons from PS animals relative to those from controls (two-way ANOVA, main effect of PS: $\left.\mathrm{F}_{1,148}=4.63, P<0.05\right)$, with a mean group difference of $1.15 \mathrm{mV}$ across all ages and a maximum group difference of $3.04 \pm 1.08 \mathrm{mV}$ at P14 $(-58.46 \pm 0.65 \mathrm{mV}$ for controls and $-61.50 \pm 0.89 \mathrm{mV}$ for PS pups).

PS also influenced the maturation of action potential (AP) waveform in the BLA (Figure $3 \mathrm{~b}$ ). We previously reported that, throughout the first postnatal month in BLA principal neurons of typically developing rats, AP threshold becomes more hyperpolarized by $5-10 \mathrm{mV}$, APs become $\sim 40 \%$ faster, and a fast-afterhyperpolarization (fAHP) emerges (Ehrlich et al, 2012). In the present study, AP threshold also became significantly more hyperpolarized with age, from $-31.76 \mathrm{mV}$ in P10 controls to a mature value of $-39.24 \mathrm{mV}$ in P21 controls (two-way ANOVA, main effect of age: $\mathrm{F}_{5,162}=50.29$, $P<0.0001 ; 9 \leqslant n \leqslant 19$ ). In offspring of dams exposed to PS, AP threshold was more hyperpolarized relative to controls across all ages (Figure $3 c_{1}$; two-way ANOVA, main effect of PS: $\left.\mathrm{F}_{1,162}=8.41, P<0.01\right)$ and by at least $0.99 \mathrm{mV}$ at every time point but P28. Although AP threshold was more hyperpolarized in PS animals, APs were taller in this population (Figure $3 c_{2}$; main effect of PS: $F_{1,165}=5.23, P<0.05$ ), with a mean group difference of $4.02 \mathrm{mV}$ across all ages and a maximum group difference of $9.65 \pm 3.78 \mathrm{mV}$ at P17 $(61.75 \pm 2.45 \mathrm{mV}$ for controls and $71.40 \pm 1.76 \mathrm{mV}$ for PS pups).

Unlike AP threshold and amplitude, AP kinetics did not change due to PS. With age, AP half-width decreased by $\sim 40 \%$, for example, from $1.22 \pm 0.06 \mathrm{~ms}$ in controls at P10 to $0.75 \pm 0.04 \mathrm{~ms}$ in controls at P28 (Figure $3 \mathrm{c}_{3}$; two-way ANOVA, main effect of age: $\left.\mathrm{F}_{5,164}=39.90, P<0.0001 ; 9 \leqslant n \leqslant 19\right)$. AP half-width was not influenced by PS at any time point (main effect of PS: $\mathrm{F}_{1,164}=3.54, P>0.05$ ). AP $10-90 \%$ rise time decreased by $\sim 30 \%$ with age, for example, from 

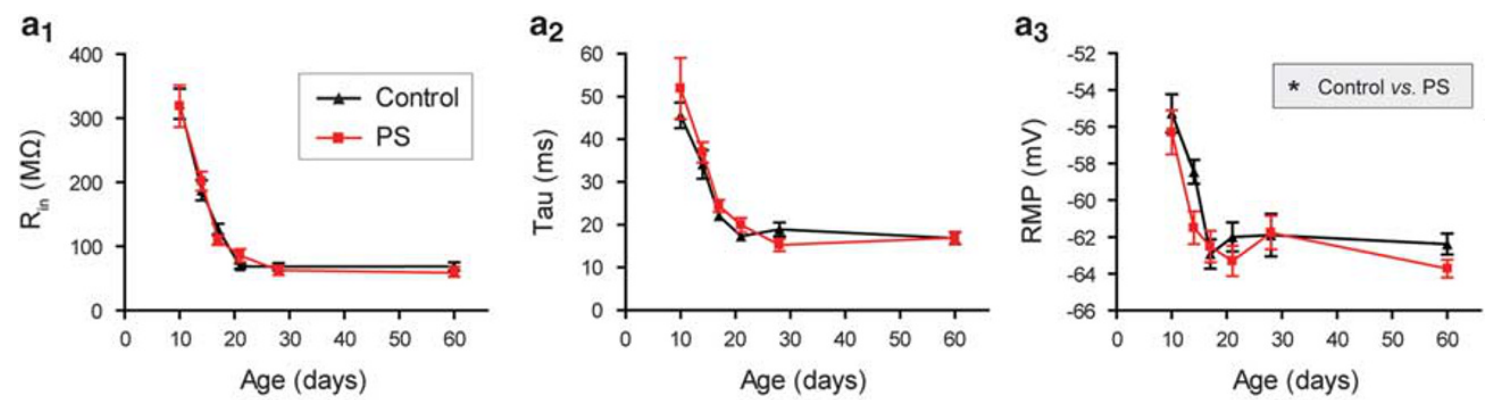

b
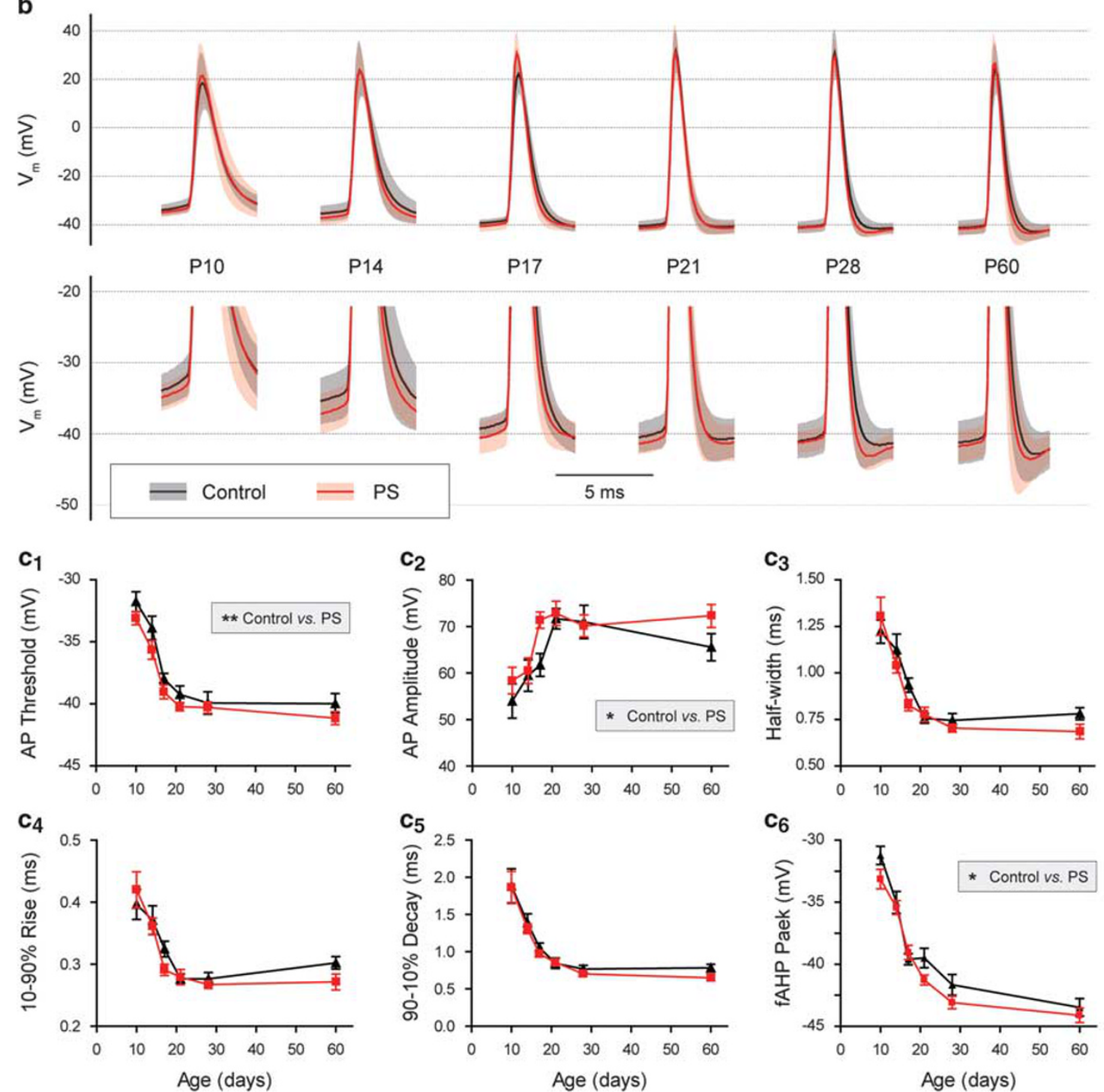

Figure 3 Effects of PS on development of intrinsic membrane properties of BLA neurons. $\left(a_{1}, a_{2}\right)$ There was no difference in the age-dependent changes to input resistance $\left(R_{\mathrm{in}}, \mathrm{a}_{1}\right)$ or membrane time constant $\left(\mathrm{a}_{2}\right)$ in PS rats compared with controls. $\left(a_{3}\right)$ Resting membrane potential (RMP) was significantly more hyperpolarized across all ages in PS rats compared with controls. (b) Action potential (AP) waveforms, depicted as means (solid lines) and SD (shaded bands), are shown for BLA principal neurons across postnatal development. The same data are shown at two resolutions to depict the full waveform (top) and the AP threshold and fast afterhyperpolarization (fAHP, bottom). ( $\left.c_{1}\right)$ AP threshold was significantly more hyperpolarized across all ages in PS animals. ( $C_{2}$ ) APs were significantly taller in PS animals. $\left(c_{3}-c_{5}\right)$ No effect of PS was observed on AP kinetics, in terms of half-width $\left(c_{3}\right), 10-90 \%$ rise time ( $\left.c_{4}\right)$, and $90-10 \%$ decay time $\left(c_{5}\right) .\left(c_{6}\right)$ The voltage at the peak of the fAHP was significantly more hyperpolarized in PS animals relative to controls.

$0.40 \pm 0.02 \mathrm{~ms}$ in controls at P10 to $0.28 \pm 0.01 \mathrm{~ms}$ in controls at P21 (Figure 3c ; two-way ANOVA, main effect of age: $\mathrm{F}_{5,165}=25.02, P<0.0001$ ), but exhibited no effect of PS (main effect of PS: $\left.\mathrm{F}_{1,165}=2.11, P>0.05\right)$. Similarly, AP $90-10 \%$ decay time decreased more than twofold with age, from $1.88 \pm 0.23 \mathrm{~ms}$ in controls at P10 to $0.77 \pm 0.05 \mathrm{~ms}$ in controls at P28 (Figure $3 c_{5}$; two-way ANOVA, main effect of age: $\left.\mathrm{F}_{5,164}=55.67, P<0.0001\right)$. AP decay times tended to be faster 
in PS offspring relative to controls at P28 $(0.77 \pm 0.05 \mathrm{~ms}$ in controls vs $0.70 \pm 0.10 \mathrm{~ms}$ in PS offspring) and P60 $(0.79 \pm 0.03 \mathrm{~ms}$ in controls $v s 0.66 \pm 0.05 \mathrm{~ms}$ in PS offspring), but the effect was not significant (main effect of PS: $\left.\mathrm{F}_{1,164}=2.97, P>0.05\right)$.

We also assessed the effect of PS on the strength of the fAHP throughout development (Figure $3 b$ and $c_{6}$ ). The fAHP was more pronounced in neurons from PS animals relative to controls, with a more hyperpolarized membrane potential at the peak of the fAHP (two-way ANOVA, main effect of PS: $\left.\mathrm{F}_{1,153}=5.73, P<0.05 ; 9 \leqslant n \leqslant 19\right)$. The effect of PS was most pronounced at P21 $(-39.48 \pm 0.80 \mathrm{mV}$ in controls vs $-41.25 \pm 0.40 \mathrm{mV}$ in PS offspring) and P28 $(-41.65 \pm 0.84 \mathrm{mV}$ in controls $v s-43.08 \pm 0.51 \mathrm{mV}$ in PS offspring).

The effects of PS on the AHP were not restricted to the fAHP. The medium AHP (mAHP), lasting several hundred milliseconds after the AP, was also strengthened throughout development by PS (Figure 4). Although the peak voltage of the mAHP was unaffected by PS, showed no clear effect of age, and typically fell between -47 and $-50 \mathrm{mV}$, the duration of the mAHP was increased by PS (Figure 4a and $b$ ). The duration from the onset of the AP to the peak of the mAHP was significantly greater in neurons from PS animals than in those from controls (two-way ANOVA, main effect of PS: $\left.\mathrm{F}_{1,100}=4.92, P<0.05 ; 5 \leqslant n \leqslant 17\right)$, with a mean group difference of $9.04 \mathrm{~ms}$. The effect of PS on mAHP peak time was most pronounced before P28, and the maximum group difference of $21.73 \pm 10.39$ ms was observed at P21 (50.61 $\pm 3.68 \mathrm{~ms}$ for controls $v s 72.34 \pm 6.16 \mathrm{~ms}$ for PS pups). There was no significant effect of age or PS on the post-burst slow AHP (sAHP) measured for the two different burst intensities (Figure $4 c ; 6 \leqslant n \leqslant 17$ ). However, the mean sAHP was consistently larger at P21, P28, and P60 in neurons from PS animals relative to controls.

\section{PS Reduced the Excitability of Adult BLA Neurons}

We previously reported that BLA principal neuron excitability decreases across the first postnatal month, based on the production of APs in response to direct depolarizing current injection (Ehrlich et al, 2012). Here we show that PS reduced the excitability of these neurons in adulthood (Figure 5). In control offspring, the rheobase, or the minimum current injection required to elicit a single AP, increased more than sixfold before reaching the mature value at P21 (Figure 5a; $164.17 \pm 13.52 \mathrm{pA}$ for P21 controls $v s 24.38 \pm 4.38 \mathrm{pA}$ for P10 controls; two-way ANOVA, main effect of age: $\left.\mathrm{F}_{5,140}=115.6, P<0.0001 ; 8 \leqslant n \leqslant 17\right)$. In contrast, the rheobase in PS neurons did not plateau at P21, but continued to increase until P60 (interaction effect: $\mathrm{F}_{5,140}=4.45, P<0.001$ ). The rheobase was nearly twice as large in PS neurons relative to controls at P60 $(165.45 \pm 14.36 \mathrm{pA}$ for controls $v s 317.14 \pm 27.58 \mathrm{pA}$ for PS adults; Bonferroni post-test, $P<0.0001$; main effect of PS: $\mathrm{F}_{1,140}=3.89, P=0.051$ ).

Despite increasing the rheobase of BLA neurons in adult offspring, PS did not influence the production of APs at high frequencies. The maximum number of APs produced by BLA principal neurons during a 1-s train typically increased with age, from $24.82 \pm 2.99$ APs in controls at P10 to the mature value of $40.10 \pm 2.66 \mathrm{APs}$ in controls at P21 (Figure 5b; two-way ANOVA, main effect of age: $\mathrm{F}_{5,139}=11.30, P<0.0001$; a
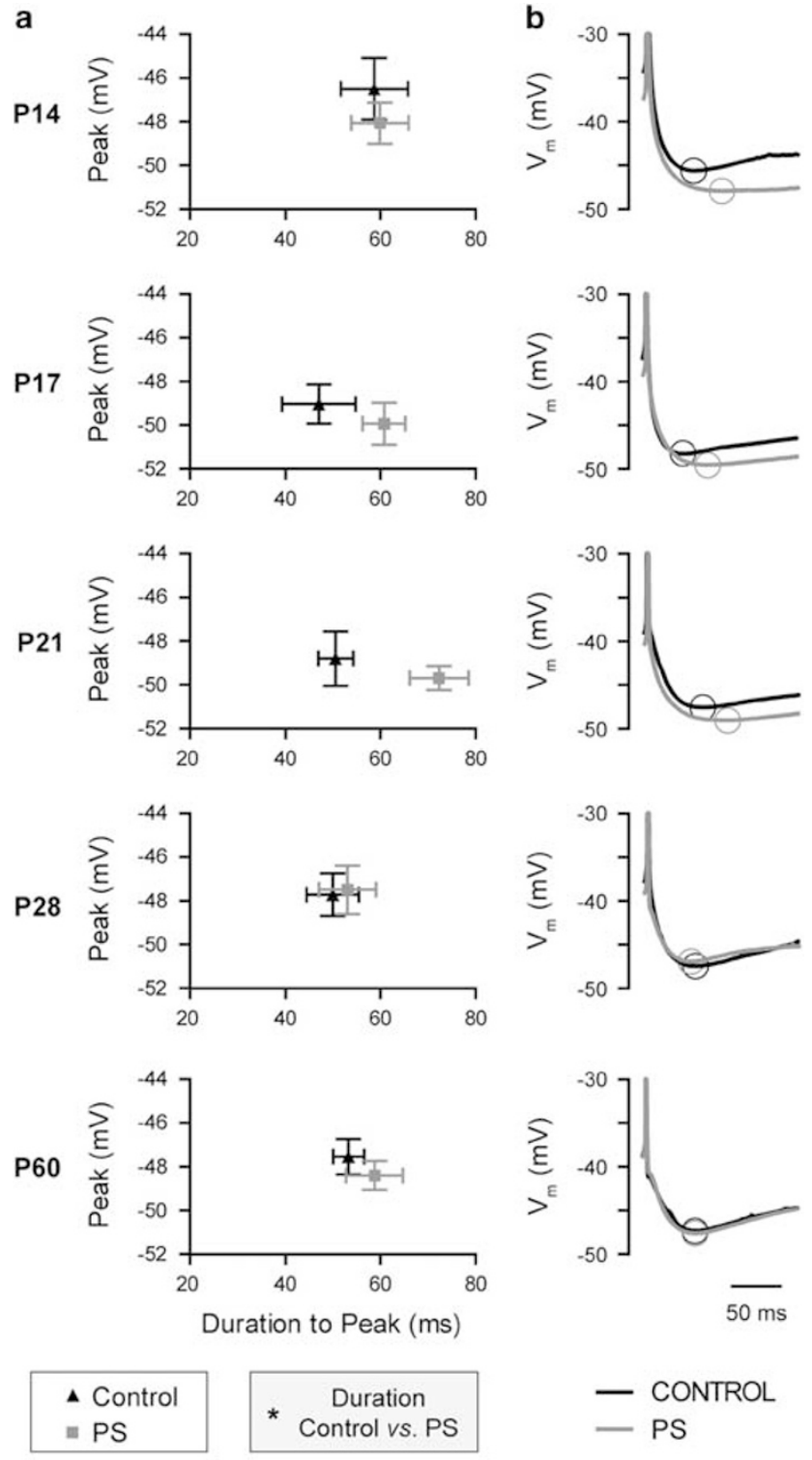

C
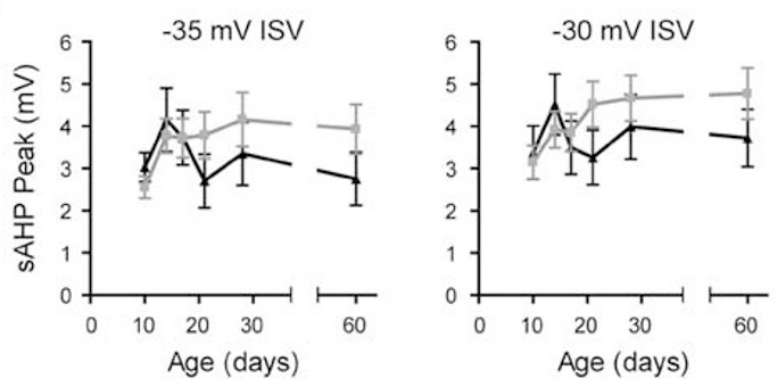

Figure 4 PS enhanced the medium afterhyperpolarization (mAHP) of BLA principal neurons. (a) The voltage at the peak of the MAHP and duration from action potential initiation to MAHP peak are plotted for PS and control neurons at each time point as mean \pm SEM. Duration to mAHP was significantly greater across all ages in PS offspring relative to controls. (b) Average traces illustrating group mAHP waveforms for PS and control neurons at every time point. A circle highlights the peak of each trace. (c) Peak voltage deflections (relative to action potential threshold) of slow AHPs (sAHPs) elicited by I-s bursts of action potentials are plotted for PS and control offspring at every time point. Traces were included in a plot if the mean inter-spike voltage (ISV) was within I $\mathrm{mV}$ of $-35 \mathrm{mV}$ (left) or $-30 \mathrm{mV}$ (right). 
a

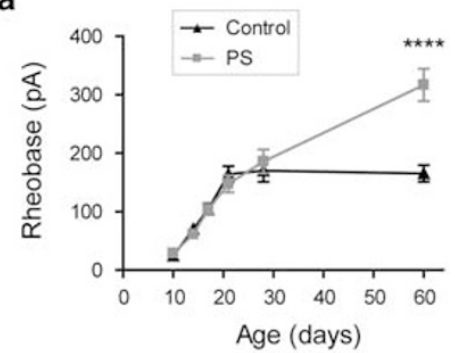

b

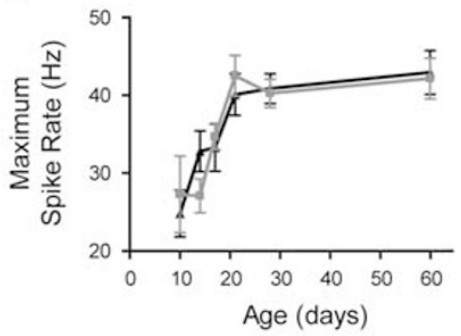

C

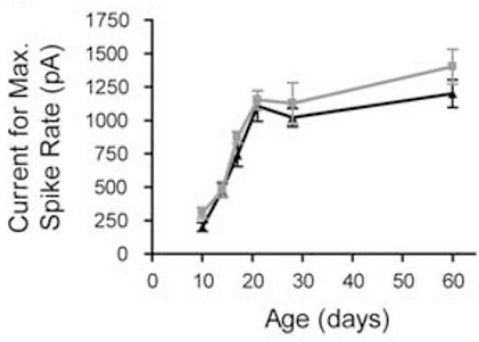

d
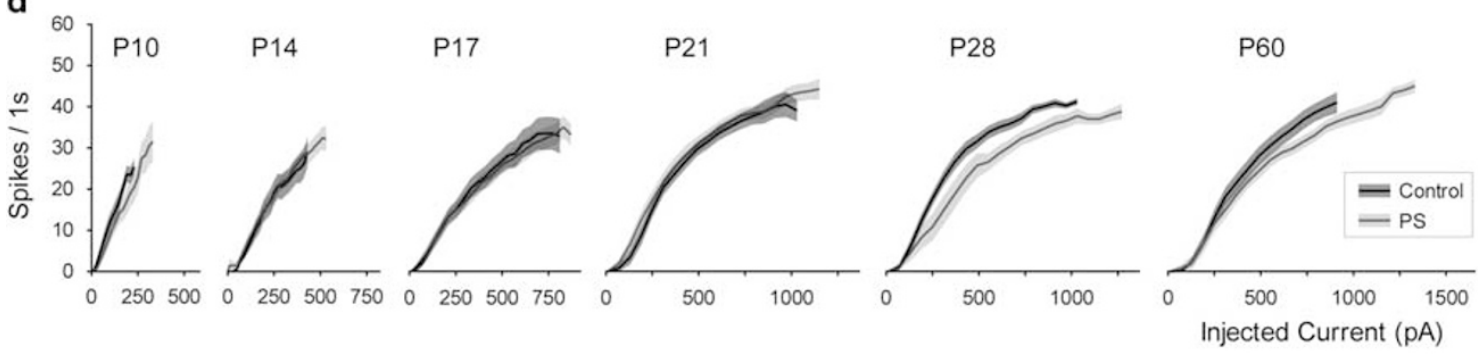

e

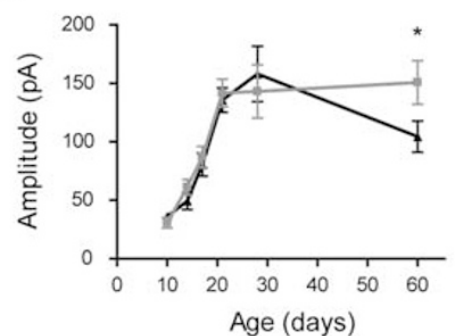

f

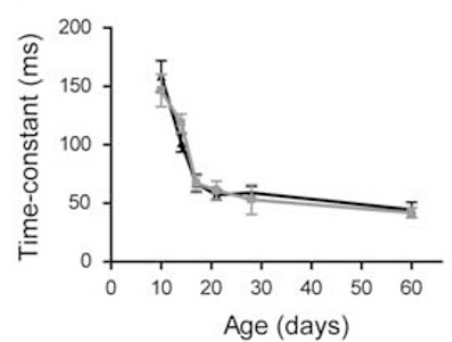

g

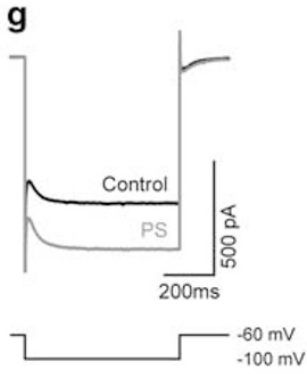

h

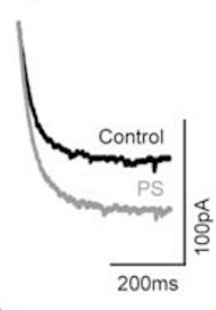

Figure 5 PS reduced BLA principal neuron excitability in adulthood but not infancy. (a) The rheobase was significantly larger at P60 in neurons from PS offspring relative to controls. (b) No effect of PS was observed on maximum spike rate. (c) The minimum current injection required to elicit the maximum spike rate increased with age but was not affected by PS. (d) F-I curves are plotted as mean (lines) and SEM (shaded bands) of the spike output for a given current injection, for both PS and control neurons. (e) H-current $\left(I_{h}\right)$ was measured from responses in voltage-clamp to hyperpolarizing steps from - 60 to $-100 \mathrm{mV}$, and the steady-state amplitude is plotted for PS and control offspring at every time point. I amplitude was significantly greater in PS animals at P60. (f) No effect of PS was observed on the time constant of activation for $l_{h}$. $(g, h)$ Average voltage-clamp waveforms of responses to the hyperpolarizing step are depicted for control and PS neurons at P60 (g), and are baseline-adjusted, magnified, and cropped to illustrate the effect of PS on $I_{h}(h)$.

$7 \leqslant n \leqslant 19)$. The maximum number of spikes was unaffected by PS (main effect of PS: $F_{1,139}=0.007, P>0.05$ ). Similarly, there was an increase with age of the minimum intensity of current injection required to elicit $\mathrm{APs}$ at that maximum rate (Figure $5 c$; main effect of age: $F_{5,139}=41.50, P<0.0001$ ). For example, BLA principal neurons from control pups at P10 required $200.91 \pm 29.18 \mathrm{pA}$ to fire those 24.82 APs, on average, whereas neurons at P21 required $1108.00 \pm 115.26 \mathrm{pA}$ to fire those 40.10 APs, on average. There was no significant effect of PS on this current injection, although PS neurons at P60 tended to require more current to fire maximally than age-matched controls $(1201.43 \pm 103.32 \mathrm{pA}$ for controls $v s 1403.85 \pm 129.64 \mathrm{pA}$ for PS adults; main effect of PS: $\left.\mathrm{F}_{1,139}=3.71, P=0.056\right)$. This trend is also evident from F-I curves, which illustrate the weaker AP output for a given current input for PS neurons relative to controls at P28 and P60 (Figure 5d).

\section{PS Enhanced H-current of Adult BLA Neurons}

We previously reported a developmental enhancement of H-current $\left(I_{h}\right)$ in BLA principal neurons, with a sevenfold increase in amplitude and nearly an order of magnitude decrease in activation time constant (Ehrlich et al, 2012). Here we contrasted the maturation of this current in PS and control offspring. We found the similar developmental trajectories of $I_{\mathrm{h}}$ expression in infancy for neurons from PS and control offspring, but a significant increase in $I_{\mathrm{h}}$ amplitude in PS adults relative to controls (Figure 5e-h; $104.4 \pm 13.3 \mathrm{pA}$ for $\mathrm{P} 60$ controls $v s 150.7 \pm 18.6 \mathrm{pA}$ for $\mathrm{P} 60$ PS offspring; Bonferroni post-test, $P<0.05$; two-way ANOVA, main effect of age: $F_{5,225}=30.84, P<0.0001$; $14 \leqslant n \leqslant 26$ ). There was no effect of PS on the developmental trajectory of $I_{\mathrm{h}}$ activation kinetics (Figure 5f; two-way ANOVA, main effect of age: $\mathrm{F}_{5,185}=45.71, P<0.0001$; main effect of PS: $\left.F_{1,185}=0.000, P>0.05\right)$.

\section{DISCUSSION}

We performed the first characterization of PS effects on functional maturation of the amygdala, identifying a constellation of changes to neurophysiology that varied in age of onset and duration (summarized in Figure 6). Decreases in 


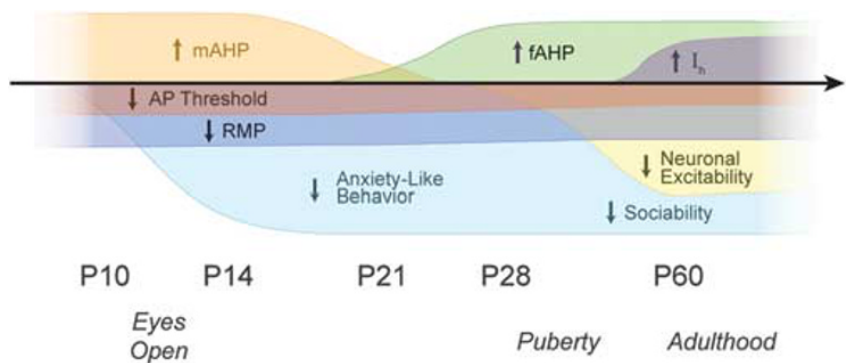

Figure 6 Summary of PS effects on the development of offspring behavior and BLA electrophysiology. The effects of PS are represented as approximate trajectories and include: more hyperpolarized action potential (AP) threshold and resting membrane potential (RMP) across development, strengthened medium AHP (mAHP) until around P2I, strengthened fast AHP (fAHP) from P2I onward, reduced anxiety-like behavior as early in infancy and adulthood, reduced sociability in adulthood, and reduced neuronal excitability and increased $\mathrm{H}$-current $\left(I_{h}\right)$ in adulthood.

amygdala neuron excitability long after PS exposure corresponded with reductions in socioemotional behavior across postnatal development. We observed PS-dependent changes to intrinsic membrane properties, including AP threshold, AP amplitude, and RMP as early as P10, and found reduced neuronal excitability and alterations to the voltage-gated current, $I_{\mathrm{h}}$, that emerged in adolescence. By characterizing developmental trajectories, we found that PS elicited behavioral changes as early as infancy and had age-specific effects on amygdala electrophysiology.

PS reduced offspring anxiety-like behavior both in infancy and adulthood. In adult offspring, PS increased the time spent on the open arms and head dips produced on the EPM and reduced defecation in the open-field test. A precedent exists for anxiolytic effects of PS in rodents (Estanislau and Morato, 2006; Mairesse et al, 2007), but most studies have found enhanced anxiety in offspring (Darnaudery and Maccari, 2008). No study has identified the critical aspects of prenatal stressors that determine the direction of effects on offspring anxiety, and no consistent differences exist in the stress paradigms employed in studies finding anxiolytic and anxiogenic effects. Given the potency and unpredictable nature of the stressor employed in the present study, we propose stronger stress responses in dams may be more likely to reduce offspring anxiety. However, many characteristics of stressors likely interact, as the impact of PS on offspring emotional behavior is sensitive to stress predictability (Fride and Weinstock, 1984; Richardson et al, 2006), timing (Barlow et al, 1978), and intensity (Mabandla et al, 2008). The effects on amygdala function observed here, similar to those on emotional behavior, may be particular to this PS paradigm and the male offspring.

The reduction of isolation-induced USVs at P17 also constituted an anxiolytic effect of PS (Morgan et al, 1999; Hofer et al, 2002), consistent with a previous study that found PS suppresses USVs at P14 (Morgan et al, 1999). The pitch of USVs from control pups decreased with age, as previously reported (Kromkhun et al, 2013), but increased with age in PS pups, further suggesting atypical development following PS. Isolation-induced USVs are inherently social (Hofer et al, 2002; Harmon et al, 2008), and reductions of USVs have been found in rodent models of ASDs (Crawley, 2007; Higashida et al, 2011). Diminished USVs in rat pups are suggested to model the lack of crying observed in some children with ASDs (Crawley, 2007). The early deficit in USV production may therefore reflect social dysfunction that persists into adulthood.

PS reduced sociability in adulthood, as PS offspring spent less time than controls interacting with a CON. PS offspring did not differ from controls in the social novelty test, exhibiting the same preference for a novel CON over a familiar one. Given the anxiolytic effect of PS in the EPM, we speculate the reduced sociability is driven by a change in social motivation and is not due to the heightened social anxiety.

The behavioral deficits in PS offspring concurred with atypical development of BLA principal neuron electrophysiology. Certain effects on intrinsic membrane properties, including a shift of RMP and AP threshold to more hyperpolarized potentials and an increase in AP amplitude, persisted throughout infancy and adulthood. In these cases, neurons from PS offspring exhibited a developmental trajectory parallel to normative development, with RMP and AP threshold becoming more hyperpolarized and AP amplitude increasing with age (Ehrlich et al, 2012). The shift of RMP and AP threshold due to PS was $\sim 1-2 \mathrm{mV}$ on average at each time point. These two changes may have opposing effects on neuronal excitability, as we found no net effect of PS on neuronal excitability in infancy. However, the effect of PS on AP threshold may serve to shift the operational subthreshold voltage range of these neurons, potentially reducing the influence on AP generation of currents that are normally active near threshold, such as $I_{\mathrm{T}}$ and $I_{\mathrm{A}}$. Such a shift could influence the production of subthreshold membrane potential oscillations, which are important for the mature function of BLA principal neurons (Ryan et al, 2012). Corroborating the effect on AP threshold, AP amplitude was also increased due to PS, possibly reflecting greater expression of voltage-gated sodium currents mediating the AP.

PS also influenced AHPs of developing BLA principal neurons. PS increased the amplitude of the fAHP in offspring from P21 to P60. A larger fAHP should promote repetitive spiking, but AP threshold or the mAHP may have obscured such an effect. The mAHP was slower in PS offspring relative to controls, most prominently before P28. At P21, 28, and 60, PS increased the post-burst sAHP, although the trend was not significant. Such an effect may contribute to the reduced excitability observed in adolescence and adulthood.

PS increased the rheobase nearly twofold at P60 and rightshifted F-I curves at P28 and 60. Given that PS and control neurons at P60 exhibited comparable $R_{\text {in }}$ and a similar voltage difference between the RMP and AP threshold, comparable amounts of depolarizing current should be required to drive an $\mathrm{AP}$. This discrepancy implies changes to voltage-gated currents that are activated or inactivated between RMP and the AP threshold. We also observed an increase in $I_{\mathrm{h}}$ amplitude by PS selectively at P60. This increase in $I_{\mathrm{h}}$ amplitude may contribute to the increase in the rheobase. Greater $I_{\mathrm{h}}$ in P60 neurons from PS offspring would predict a more depolarized RMP, but the opposite effect was observed, suggesting compensation via the loss of other inward currents or enhancement of outward currents. As neurons are depolarized from RMP towards AP threshold, $I_{\mathrm{h}}$ deactivation should reveal the effect of compensatory currents to oppose AP generation. 
Amygdala activity contributes to social and emotional behaviors, and the reduction in amygdala neuron excitability following PS may simultaneously inhibit sociability and anxiety-like behavior. The fact that our PS paradigm caused social deficits while reducing anxiety suggests PS may act distinctly on various symptom dimensions, consistent with the variety of disorders and behavioral phenotypes linked to PS. The social deficits observed here suggest our PS offspring model some aspects of neurodevelopmental disorders such as ASDs and schizophrenia. In support of this notion, amygdala responses to social and emotional stimuli are dampened in schizophrenia patients (Rasetti et al, 2009), and treatments that remediate this activity deficit are more effective (Hooker et al, 2013). The anxiolytic effect of PS may reflect generally dampened emotionality, and may in fact be parsimonious with reduced sociability.

An alternative interpretation of the anxiolytic effects is that PS promotes resilience in offspring. A recent study compared BLA principal neurons of rats inherently resilient or vulnerable to stress (Hetzel and Rosenkranz, 2014). Interestingly, BLA neurons from resilient rats shared a number of properties with neurons from adult PS offspring in the present study. Neurons from resilient rats had shallower F-I curves and larger $I_{\mathrm{h}}$ and AHP than neurons from vulnerable rats, and neurons from our PS adults had comparable differences relative to control adults.

The late effects of PS on BLA neuron excitability may occur downstream of earlier deficits, and identifying the progression of PS-induced developmental changes will provide potential targets for intervention in the progression from early-life stress to psychiatric disease. The steps linking PS to altered amygdala physiology may partially occur postnatally, as early postnatal manipulations can also influence the development of emotional behavior and amygdala function (Callaghan and Richardson, 2013; Rincon-Cortes and Sullivan, 2014). More specifically, PS may indirectly influence amygdala physiology of offspring via direct effects on behavior of the dam, as offspring emotion is highly sensitive to maternal behavior (Zhang et al, 2006). Our PS paradigm acutely enhanced dam freezing in the shock context, and behavioral alterations may persist during rearing. Fostering offspring of control dams to stressed dams introduces some mild effects of PS, suggesting the effects of PS are conferred both directly at the time of stress and indirectly via maternal behavior (Barlow et al, 1978). Future studies will be required to identify the direct mechanisms by which PS influences amygdala neurophysiology in offspring.

\section{FUNDING AND DISCLOSURE}

This work was funded by the following grants from the National Institutes of Health: MH 069852 to DGR, base grant RR 00165 to the Yerkes National Primate Research center, and MH 090729 to DEE. The authors declare no conflict of interest.

\section{ACKNOWLEDGMENTS}

We thank Dr Shannon Gourley for feedback during the preparation of this manuscript, and Dr Steve Ryan, Dr Teresa Madsen, and Wei Liu for assistance with data analysis.

\section{REFERENCES}

Amaral DG, Schumann CM, Nordahl CW (2008). Neuroanatomy of autism. Trends Neurosci 31: 137-145.

Barlow SM, Knight AF, Sullivan FM (1978). Delay in postnatal growth and development of offspring produced by maternal restraint stress during pregnancy in the rat. Teratology 18: 211-218.

Bayer SA, Altman J, Russo RJ, Zhang X (1993). Timetables of neurogenesis in the human brain based on experimentally determined patterns in the rat. Neurotoxicology 14: 83-144.

Benes FM (2010). Amygdalocortical circuitry in schizophrenia: from circuits to molecules. Neuropsychopharmacology 35: 239-257.

Berdel B, Morys J, Maciejewska B, Dziewiatkowski J (1997a). Volume and topographical changes of the basolateral complex during the development of the rat's amygdaloid body. Folia Morphol (Warsz) 56: 1-11.

Berdel B, Morys J, Maciejewska B (1997b). Neuronal changes in the basolateral complex during development of the amygdala of the rat. Int J Dev Neurosci 15: 755-765.

Blackford JU, Pine DS (2012). Neural substrates of childhood anxiety disorders: a review of neuroimaging findings. Child Adoles Psychiatr Clin N Am 21: 501-525.

Callaghan BL, Richardson R (2013). Early experiences and the development of emotional learning systems in rats. Biol Mood Anxiety Disord 3: 8.

Callaghan BL, Graham BM, Li S, Richardson R (2013). From resilience to vulnerability: mechanistic insights into the effects of stress on transitions in critical period plasticity. Front Psychiatry 4: 90.

Crawley JN (2007). Mouse behavioral assays relevant to the symptoms of autism. Brain Pathol 17: 448-459.

Darnaudery M, Maccari S (2008). Epigenetic programming of the stress response in male and female rats by prenatal restraint stress. Brain Res Rev 57: 571-585.

Deoni SC, Mercure E, Blasi A, Gasston D, Thomson A, Johnson M et al (2011). Mapping infant brain myelination with magnetic resonance imaging. J Neurosci 31: 784-791.

Ehrlich DE, Ryan SJ, Rainnie DG (2012). Postnatal development of electrophysiological properties of principal neurons in the rat basolateral amygdala. J Physiol 590: 4819-4838.

Ehrlich DE, Ryan SJ, Hazra R, Guo JD, Rainnie DG (2013). Postnatal maturation of GABAergic transmission in the rat basolateral amygdala. J Neurophysiol 110: 926-941.

Estanislau C, Morato S (2006). Behavior ontogeny in the elevated plus-maze: prenatal stress effects. Int J Dev Neurosci 24: 255-262.

Felix-Ortiz AC, Tye KM (2014). Amygdala inputs to the ventral hippocampus bidirectionally modulate social behavior. J Neurosci 34: 586-595.

Fride E, Weinstock M (1984). The effects of prenatal exposure to predictable or unpredictable stress on early development in the rat. Dev Psychobiol 17: 651-660.

Giedd JN, Vaituzis AC, Hamburger SD, Lange N, Rajapakse JC, Kaysen D et al (1996). Quantitative MRI of the temporal lobe, amygdala, and hippocampus in normal human development: ages 4-18 years. J Com Neurol 366: 223-230.

Harmon KM, Cromwell HC, Burgdorf J, Moskal JR, Brudzynski SM, Kroes RA et al (2008). Rats selectively bred for low levels of $50 \mathrm{kHz}$ ultrasonic vocalizations exhibit alterations in early social motivation. Dev Psychobiol 50: 322-331.

Hazra R, Guo JD, Dabrowska J, Rainnie DG (2012). Differential distribution of serotonin receptor subtypes in BNST(ALG) neurons: modulation by unpredictable shock stress. Neuroscience 225: 9-21.

Hetzel A, Rosenkranz JA (2014). Distinct effects of repeated restraint stress on basolateral amygdala neuronal membrane 
properties in resilient adolescent and adult rats. Neuropsychopharmacology 39: 2114-2130.

Higashida H, Yokoyama S, Munesue T, Kikuchi M, Minabe Y, Lopatina O (2011). CD38 gene knockout juvenile mice: a model of oxytocin signal defects in autism. Biol Pharm Bull 34: 1369-1372.

Hofer MA, Shair HN, Brunelli SA (2002). Ultrasonic vocalizations in rat and mouse pups. Curr Protoc Neurosci 8(8): 14.

Hooker CI, Bruce L, Fisher M, Verosky SC, Miyakawa A, D'Esposito $M$ et al (2013). The influence of combined cognitive plus social-cognitive training on amygdala response during face emotion recognition in schizophrenia. Psychiatry Res 213: 99-107.

Khashan AS, Abel KM, McNamee R, Pedersen MG, Webb RT, Baker PN et al (2008). Higher risk of offspring schizophrenia following antenatal maternal exposure to severe adverse life events. Arch Gen Psychiatry 65: 146-152.

King EC, Pattwell SS, Glatt CE, Lee FS (2014). Sensitive periods in fear learning and memory. Stress 17: 13-21.

Kinney DK, Munir KM, Crowley DJ, Miller AM (2008). Prenatal stress and risk for autism. Neurosci Biobehav Rev 32: 1519-1532.

Knutson B, Burgdorf J, Panksepp J (2002). Ultrasonic vocalizations as indices of affective state in rats. Psychol Bull 128: 961-977.

Kraszpulski M, Dickerson PA, Salm AK (2006). Prenatal stress affects the developmental trajectory of the rat amygdala. Stress 9: $85-95$.

Kromkhun P, Katou M, Hashimoto H, Terada M, Moon C, Saito TR (2013). Quantitative and qualitative analysis of rat pup ultrasonic vocalization sounds induced by a hypothermic stimulus. Lab Anim Res 29: 77-83.

Laloux C, Mairesse J, Van Camp G, Giovine A, Branchi I, Bouret S et al (2012). Anxiety-like behaviour and associated neurochemical and endocrinological alterations in male pups exposed to prenatal stress. Psychoneuroendocrinology 37: 1646-1658.

Landers MS, Sullivan RM (2012). The development and neurobiology of infant attachment and fear. Dev Neurosci 34: 101-114.

LeDoux J (2007). The amygdala. Curr Biol 17: R868-R874.

Mabandla MV, Dobson B, Johnson S, Kellaway LA, Daniels WM, Russell VA (2008). Development of a mild prenatal stress rat model to study long term effects on neural function and survival. Metab Brain Dis 23: 31-42.

Mairesse J, Viltart O, Salome N, Giuliani A, Catalani A, Casolini P et al (2007). Prenatal stress alters the negative correlation between neuronal activation in limbic regions and behavioral responses in rats exposed to high and low anxiogenic environments. Psychoneuroendocrinology 32: 765-776.

McDonald AJ (1985). Immunohistochemical identification of gamma-aminobutyric acid-containing neurons in the rat basolateral amygdala. Neurosci Lett 53: 203-207.
Monk C, Spicer J, Champagne FA (2012). Linking prenatal maternal adversity to developmental outcomes in infants: the role of epigenetic pathways. Dev Psychopathol 24: 1361-1376.

Morgan KN, Thayer JE, Frye CA (1999). Prenatal stress suppresses rat pup ultrasonic vocalization and myoclonic twitching in response to separation. Dev Psychobiol 34: 205-215.

Quirk GJ, Gehlert DR (2003). Inhibition of the amygdala: key to pathological states? Ann NY Acad Sci 985: 263-272.

Rasetti R, Mattay VS, Wiedholz LM, Kolachana BS, Hariri AR, Callicott JH et al (2009). Evidence that altered amygdala activity in schizophrenia is related to clinical state and not genetic risk. Am J Psychiatry 166: 216-225.

Richardson HN, Zorrilla EP, Mandyam CD, Rivier CL (2006). Exposure to repetitive versus varied stress during prenatal development generates two distinct anxiogenic and neuroendocrine profiles in adulthood. Endocrinology 147: 2506-2517.

Rincon-Cortes M, Sullivan RM (2014). Early Life Trauma and Attachment: Immediate and Enduring Effects on Neurobehavioral and Stress Axis Development. Front Endocrinol 5: 33.

Ronald A, Pennell CE, Whitehouse AJ (2010). Prenatal maternal stress associated with ADHD and autistic traits in early childhood. Front Psychol 1: 223.

Ryan SJ, Ehrlich DE, Jasnow AM, Daftary S, Madsen TE, Rainnie DG (2012). Spike-timing precision and neuronal synchrony are enhanced by an interaction between synaptic inhibition and membrane oscillations in the amygdala. PloS One 7: e35320.

Ryan SJ, Ehrlich DE, Rainnie DG (2014). Morphology and dendritic maturation of developing principal neurons in the rat basolateral amygdala. Brain Struct Funct e-pub ahead of print 9 November 201410.1007/s00429-014-0939-x.

Sadler TR, Nguyen PT, Yang J, Givrad TK, Mayer EA, Maarek JM et al (2011). Antenatal maternal stress alters functional brain responses in adult offspring during conditioned fear. Brain Res 1385: $163-174$.

Schulz KM, Pearson JN, Neeley EW, Berger R, Leonard S, Adams CE et al (2011). Maternal stress during pregnancy causes sex-specific alterations in offspring memory performance, social interactions, indices of anxiety, and body mass. Physiol Behav 104: 340-347.

Tottenham N, Sheridan MA (2009). A review of adversity, the amygdala and the hippocampus: a consideration of developmental timing. Front Human Neuroscience 3: 68.

Walker DL, Davis M (2008). Role of the extended amygdala in short-duration versus sustained fear: a tribute to Dr Lennart Heimer. Brain Struct Funct 213: 29-42.

Weinstock M (2008). The long-term behavioural consequences of prenatal stress. Neurosci Biobehav Rev 32: 1073-1086.

Zhang TY, Bagot R, Parent C, Nesbitt C, Bredy TW, Caldji C et al (2006). Maternal programming of defensive responses through sustained effects on gene expression. Biol Psychol 73: 72-89.

Supplementary Information accompanies the paper on the Neuropsychopharmacology website (http://www.nature.com/npp) 